\title{
Modified Glenn connection for acutely ischemic right ventricular failure reverses secondary left ventricular dysfunction
}

\author{
Mark H. Danton, MD \\ John G. Byrne, MD \\ Kathryn 0. Flores, MD \\ Michael Hsin, MBBChir \\ Jeffrey S. Martin, MD \\ Rita G. Laurence, BS \\ Lawrence H. Cohn, MD \\ Lishan Aklog, MD
}

From the Department of Cardiac Surgery, Brigham and Women's Hospital/Harvard Medical School, Boston, Mass.

Read at the Eightieth Annual Meeting of The American Association for Thoracic Surgery, Toronto, Ontario, Canada, April 30-May 3, 2000.

Received for publication May 4, 2000; revisions requested July 11, 2000; revisions received Dec 13, 2000; accepted for publication Jan 17, 2001.

Address for reprints: Lishan Aklog, MD, Division of Cardiac Surgery, Brigham and Women's Hospital/Harvard Medical School, 75 Frances St, Boston, MA 02115 (E-mail: markdanton@yahoo.com).

J Thorac Cardiovasc Surg 2001;122:80-91

Copyright (c) 2001 by The American Association for Thoracic Surgery

0022-5223/2001 \$35.00+ $0 \quad \mathbf{1 2 / 6 / 1 1 4 6 3 2}$

doi:10.1067/mtc.2001.114632
Background: Right heart failure after cardiopulmonary bypass can result in severe hemodynamic compromise with high mortality, but the underlying mechanisms remain poorly understood. After ischemia-induced right ventricular failure, alterations in the interventricular septal position decrease left ventricular compliance and limit filling but may also distort left ventricular geometry and compromise contractility and relaxation. This study investigated the effect of acute isolated right ventricular ischemia on biventricular performance and interaction and the response of subsequent right ventricular unloading by use of a modified Glenn shunt.

Methods: In 8 pigs isolated right ventricular ischemic failure was induced by means of selective coronary ligation. A modified Glenn circuit was then established by a superior vena cava-pulmonary artery connection. Ventricular performance was determined by conductance catheter-derived right ventricular pressure-volume loops and left ventricular pressure-segment length loops. Hemodynamic data at baseline, after right ventricular ischemia, and after institution of the Glenn circuit were obtained during inflow occlusion, and the load-independent contractile indices were derived.

Results: Right ventricular free-wall ischemia resulted in acute right ventricular dilation $(118 \pm 81 \mathrm{~mL}$ vs $169 \pm 70 \mathrm{~mL}, P=.0008)$ and impairment of left ventricular contractility indicated by the reduced end-systolic pressure-volume relation slope $(50.0 \pm 19 \mathrm{~mm} \mathrm{Hg} / \mathrm{mm}$ vs $18.9 \pm 8 \mathrm{~mm} \mathrm{Hg} / \mathrm{mm}, P=.002)$ and preload recruitable stroke work index slope $\left(69.6 \pm 26 \mathrm{erg} \cdot \mathrm{cm}^{-3} \cdot 10^{3}\right.$ vs $39.7 \pm 13 \mathrm{erg}$ $\left.\cdot \mathrm{cm}^{-3} \cdot 10^{3}, P=.003\right)$. In addition, left ventricular relaxation $(\tau)$ was significantly prolonged $(33.3 \pm 10 \mathrm{~ms}$ vs $53.0 \pm 16 \mathrm{~ms}, P=.012)$. Right ventricular unloading with the Glenn shunt reduced right ventricular dilation and significantly improved left ventricular contraction, end-systolic pressure-volume relation slope $(18.9 \pm 8 \mathrm{~mm} \mathrm{Hg} / \mathrm{mm}$ vs $35.8 \pm 18 \mathrm{~mm} \mathrm{Hg} / \mathrm{mm}, P=.002)$, preload recruitable stroke work index slope $\left(39.7 \pm 26 \mathrm{erg} \cdot \mathrm{cm}^{-3} \cdot 10^{3}\right.$ vs $63.0 \pm 22 \mathrm{erg}$ $\left.\cdot \mathrm{cm}^{-3} \cdot 10^{3}, P=.003\right)$, and diastolic performance $(\tau 53.0 \pm 16 \mathrm{~ms}$ vs $43.5 \pm 13$ $\mathrm{ms}, P=.001)$.

Conclusions: Right ventricular ischemia-induced dilation resulted in acute impairment of left ventricular contractility and relaxation. A modified Glenn shunt attenuated the left ventricular dysfunction by limiting right ventricular dilation and restoring left ventricular cavity geometry. 


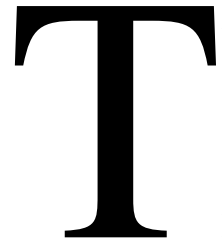

he right ventricle was once considered to act mainly as a passive conduit. This was concluded after canine studies in which right ventricular (RV) free-wall injury produced only minimal hemodynamic compromise. ${ }^{1}$ Furthermore, experience gained from the surgical treatment of tricuspid atresia demonstrated that the right ventricle can be completely ${ }^{2}$ or partially bypassed and that the circulation will be successfully maintained. Despite these findings, it is now clear that function of the right ventricle plays an integral role in the outcome of patients after myocardial infarction and many cardiac surgical procedures, including cardiac transplantation ${ }^{3}$ and left heart assist device placement. ${ }^{4}$ When it occurs in the intraoperative period, right heart failure is associated with failure to wean from cardiopulmonary bypass and the need for massive inotropic and mechanical support. Postoperative refractory RV failure has been managed with an RV assist device, pulmonary artery counterpulsation, ${ }^{5}$ or creation of an atrial septal defect, ${ }^{6}$ but success has been inconsistent, and mortality remains high.

Several mechanisms by which RV failure leads to low cardiac output have been proposed. Impaired RV contraction reduces transpulmonary blood flow and left atrial filling. In addition, RV dilation causes leftward deviation of the interventricular septum, decreasing left ventricular (LV) compliance. These effects act synergistically to compromise LV diastolic filling, which reduces stroke volume and cardiac output. ${ }^{7}$ The effect of acute RV infarction on LV systolic performance is less clear. Although some studies have shown that LV systolic function is unaffected after RV infarction, ${ }^{8}$ others have concluded that LV contractility may be impaired by distortion of ventricular geometry. ${ }^{9}$ Furthermore, the effect of RV ischemiainduced dilation on $\mathrm{LV}$ performance is attenuated by means of pericardiotomy, which acts to restore the septal position. ${ }^{9,10}$ These studies suggest that the major component of hemodynamic insult that follows RV infarction involves impairment of LV performance caused by alterations in LV cavity geometry.

Inadequate RV function after biventricular repair for complex congenital heart defects in children may be successfully managed by partial RV bypass with a bidirectional Glenn shunt, as in the one-and-a-half ventricle repair. ${ }^{11}$ Furthermore, in patients with Ebstein anomaly and dilated right ventricles, bidirectional Glenn shunts will decrease preload and effect on arrhythmias and on RV function. ${ }^{12}$ Although the mechanisms by which partial RV bypass results in hemodynamic benefit have not been studied, it is possible that reducing RV preload and limiting dilation restores LV cavity geometry and improves LV contractility.

We propose that patients with predominant RV failure after cardiopulmonary bypass might also benefit from partial bypass of the RV through a modified Glenn shunt. This will act to reduce the volume loading on the failing right ventricle, limit excessive ventricular dilation, and reduce wall stress. In addition, alterations in septal position and LV geometry will be minimized, and LV systolic and diastolic function will be preserved. To test this hypothesis, we assessed the effects of acute RV free-wall ischemia on simultaneous load-independent indices of LV and RV function in an open-chest porcine model of acute RV infarction before and after establishing a cavopulmonary shunt.

\section{Materials and Methods Surgical Preparation}

Eight Yorkshire pigs (mean weight, $45 \mathrm{~kg}$ ) of either sex were used in this study and were cared for according to the National Institutes of Health Guide for the Care and Use of Laboratory Animals. ${ }^{13}$ General anesthesia was induced with intramuscular tamsulosin (Telazol) $(5 \mathrm{mg})$ and then masked with isoflurane. Animals were intubated and ventilated with $100 \%$ oxygen, and anesthesia was maintained with $2 \%$ to $3 \%$ isoflurane. Continuous oxygen saturations and arterial blood gas analysis were performed to monitor oxygenation and optimize ventilation. Ventilation was adjusted with tidal volumes initially set at $10 \mathrm{~mL} / \mathrm{kg}$, and the ventilation rate was adjusted to maintain $\mathrm{PCO}_{2}$ values of less than $40 \mathrm{~mm} \mathrm{Hg}$, with the inflation pressures below $10 \mathrm{~cm} \mathrm{H}_{2} \mathrm{O}$. All animals received a bolus of magnesium sulfate $(2 \mathrm{mg}$ ) before instrumentation to reduce the incidence of arrhythmias. Hydration was maintained with intravenous fluids and maintained at a rate of $1 \mathrm{~mL} \cdot \mathrm{kg}^{-1} \cdot \mathrm{h}^{-1}$ with $0.9 \%$ saline solution.

Median sternotomy was performed, and the heart was supported by a pericardial cradle. A modified Glenn circuit was established by connecting the superior vena cava (SVC) to the main pulmonary artery with a short segment of donor porcine inferior vena cava (IVC), as shown in Figure 1 (designated homograft). Animals were systemically heparinized (100 U/kg), and end-to-side anastomoses were created between the homograft and the main pulmonary artery and the SVC by means of partially occluding vascular clamps. In all cases the cavopulmonary shunt was instituted without cardiopulmonary bypass, which avoided potential deleterious effects on heart and lung function.

The model of isolated RV infarction was produced by selective coronary occlusion. Typically, 4 to 6 acute marginal branches arising from the right coronary artery were encircled with a suture for subsequent ligation. RV branches supplying the interventricular septum (ie, posterior descending artery) or the inferior surface of the left ventricle were avoided to prevent injury to the LV wall or septum.

\section{Instrumentation}

An electromagnetic flow probe (model 501; Carolina Medical Electronics, King, NC) was positioned around the pulmonary artery to assess stroke volume and cardiac output.

Micromanometers (5F, model PC350; Millar Instruments, Inc, Houston, Tex) were placed in the pulmonary artery and RV and LV cavities. A fluid-filled catheter (Statham P10EZ transducer, SpectraMed) was attached to the intravenous central line for monitoring of SVC pressure. 


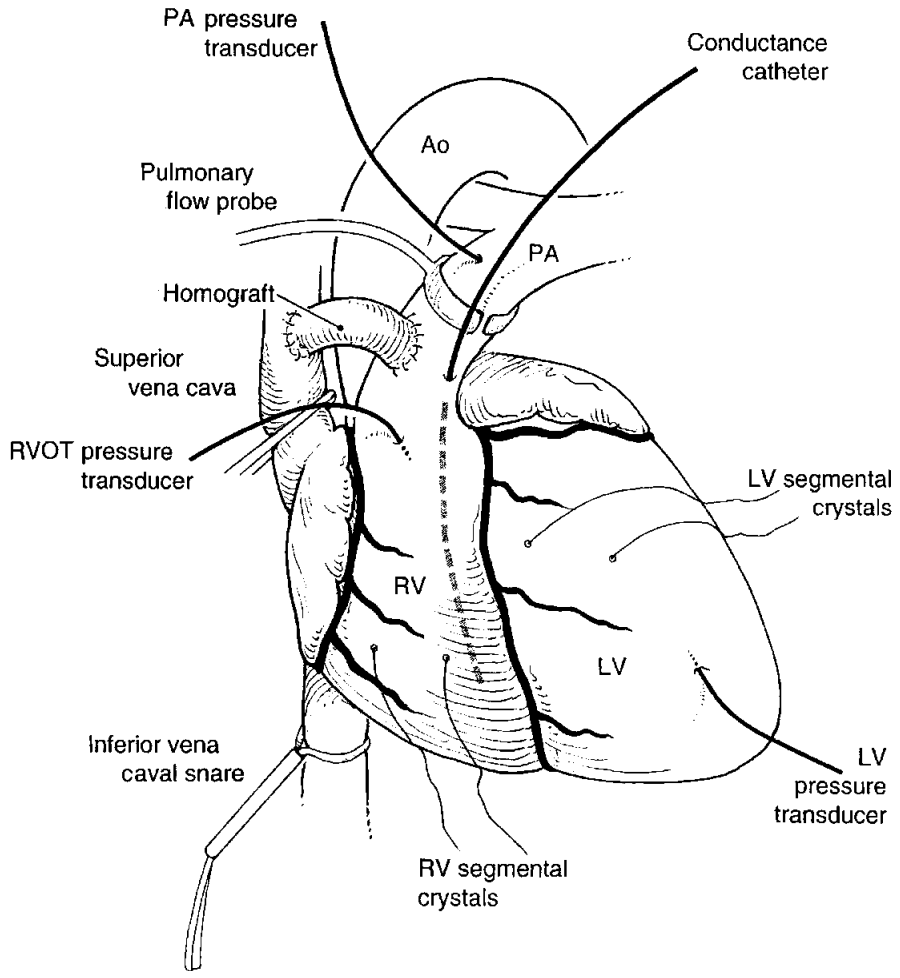

Figure 1. Animal preparation. PA, Pulmonary artery; Ao, aorta; RVOT, right ventricular outflow tract.

A 5F, 12-segment, single-field conductance catheter with 6-mm electrode spacing (Cordis Webster, Inc, a division of Johnson \& Johnson, New Brunswick, NJ) was introduced through the proximal pulmonary artery and positioned in the RV cavity. Catheter position was confirmed by using epicardial echocardiography. Final position was adjusted by inspection of the individual segment pressure-volume loops. Counterclockwise rotation of each of the 5 individual pressure-volume segments loops ensured that each was within the RV cavity.

A single pair of miniature piezoelectric crystals (Transonics Systems, Inc, Ithaca, NY) were implanted into the LV myocardium, and the ultrasound transit time provided segment length and pressure-length loop measurements. Crystals were placed in the midwall position, typically between the first and second diagonal vessels, through small stab wounds in the epicardial surface and oriented in the direction of the muscle fibers. Correct orientation of crystals was verified by analysis of the pressuresegment length loop, which should have a rectangular configuration.

The animal's temperature was maintained with a heating blanket.

\section{Data Acquisition and Analysis}

Amplified pressure, conductance, and sonomicrometer signals were sampled at $200 \mathrm{~Hz}$ and transferred through a 16-channel ana$\log$ to digital converter (model PC1 M10 16E 4, National Instruments, Austin, Tex) to a dedicated personal computer (Compaq Pentium II) by means of data acquisition software programmed in our laboratory (Labview 5.0; National Instruments).
The total conductance signal was generated and processed in a Sigma 5 DF unit. The principals of conductance method are described elsewhere. ${ }^{14}$ In brief, the conductance catheter contains a series of equally spaced electrodes designed so that the electrodes span the entire length of the ventricular cavity. A 30- $\mu \mathrm{A}, 20$ $\mathrm{kHz}$ current is generated between the proximal and distal electrodes, and the intervening electrodes record conductance between 5 electrode pairs located within the ventricle. The 5 segmental conductances are summed and converted to a time-varying volume signal. The volume, $\mathrm{V}$, of the ventricle at a given time, $\mathrm{t}$, is calculated as follows:

$$
V(t)=(1 / \alpha) L^{2} \mu[G(t)-G p]
$$

where $\mathrm{L}$ is the interelectrode distance, $\mu$ is the resistivity of blood that is measured, $\alpha$ is the gain factor derived as the ratio of conductance volume to ventricular volume derived by an alternative method, G(t) is the sum of the conductances at any time t, and Gp is the parallel conductance.

The calculation of gain factor is necessary because of nonhomogeneous spread of the electric field within the ventricular blood pool. Cumulative ejected volume determined by conductance and recorded at steady state was plotted against the integrated pulmonary flow probe signal at each time point during ventricular ejection; gain was derived as the gradient of the linear regression. Correlation coefficients of the linear regression were recorded, and coefficients of greater than 0.95 were accepted.

Parallel conductance refers to the portion of current that is conducted through the myocardium and escapes the ventricular blood 


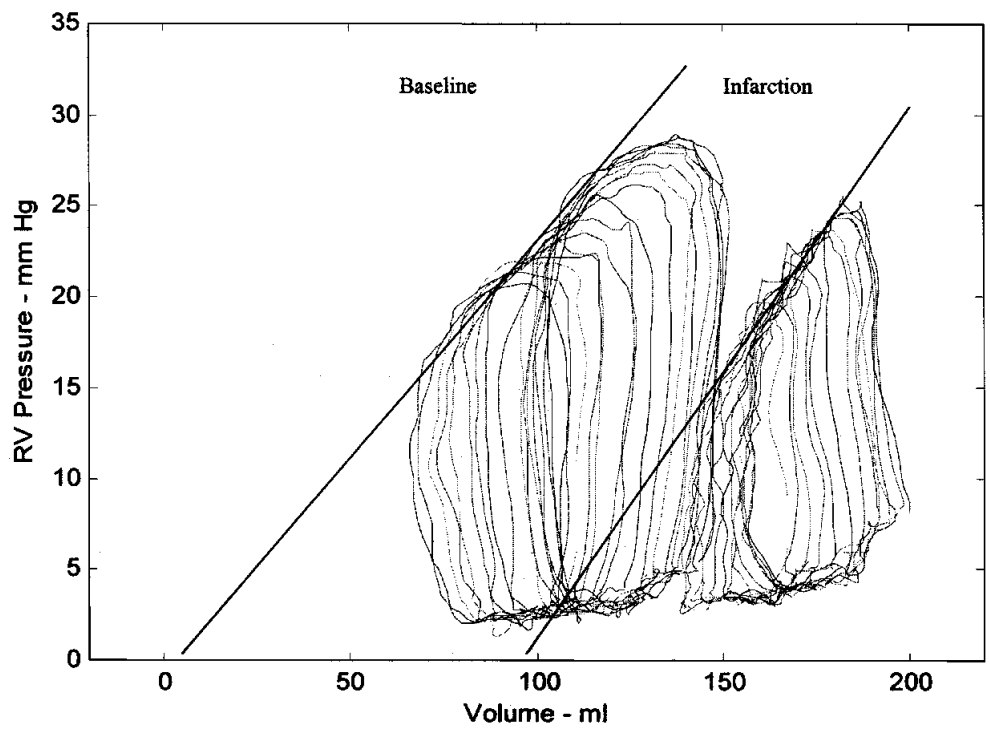

Figure 2. An example of RV pressure-volume loops before and after RV free-wall ischemia.

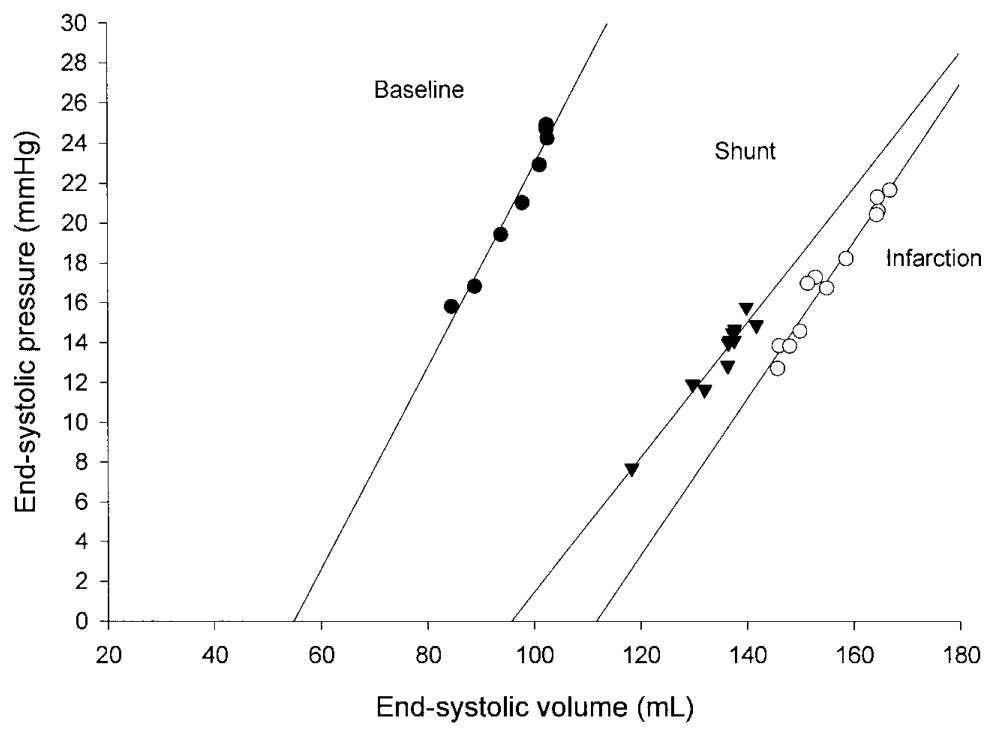

Figure 3. RV ESPVR at baseline, after ischemia, and after Glenn shunting.

pool. This was calculated by a modification of the hypertonic saline injection method developed by Baan and associates. ${ }^{14} \mathrm{~A}$ slow bolus of $10 \mathrm{~mL}$ of $10 \%$ saline solution was injected into the SVC, causing a transient increase in conductivity of blood within the ventricular cavity, without a detectable change in RV or LV pressure. For each cardiac cycle, end-systolic volumes were plotted against end-diastolic volumes. Linear regression analysis of the plots was performed, and the point of interception with the line of identity produced an estimate for parallel conductance. Correlation coefficients of the regression lines were recorded, and coefficients greater than 0.95 were accepted. For each experiment, 3 estimates of parallel conductance were made.

\section{Calculation of Systolic Function}

Calculation of indices of contractile function during transient IVC occlusion was performed with more than 8 consecutive cycles free of ventricular ectopy.

The end-systolic pressure-volume relationship (ESPVR) for each cycle under occlusion end-systole was defined as the point of maximum elastance, where elastance was calculated as follows:

$$
\mathrm{E}(\mathrm{t})=\mathrm{P}(\mathrm{t}) / \mathrm{V}(\mathrm{t})-\mathrm{V}_{0}
$$

$\mathrm{E}(\mathrm{t})$ is the time-varying elastance and $\mathrm{P}(\mathrm{t})$ and $\mathrm{V}(\mathrm{t})$ are the ventricular pressure and volume, respectively. $V_{0}$ is the theoretical unstressed ventricular volume. ESPVR was calculated by the 


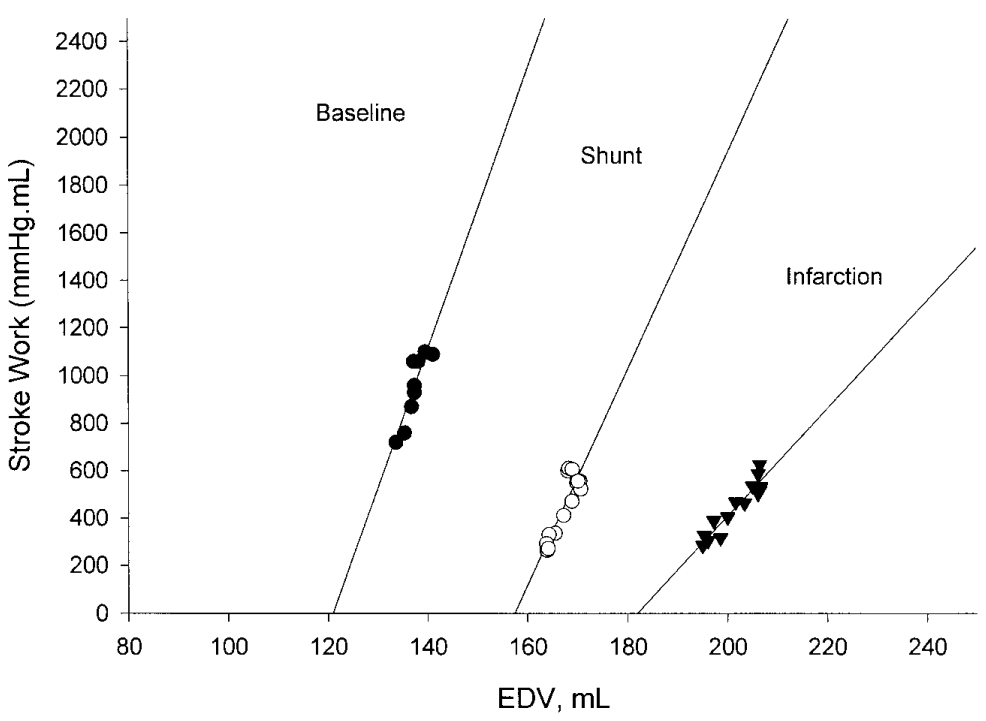

Figure 4. RV PRSW at baseline, after ischemia, and after Glenn shunting.

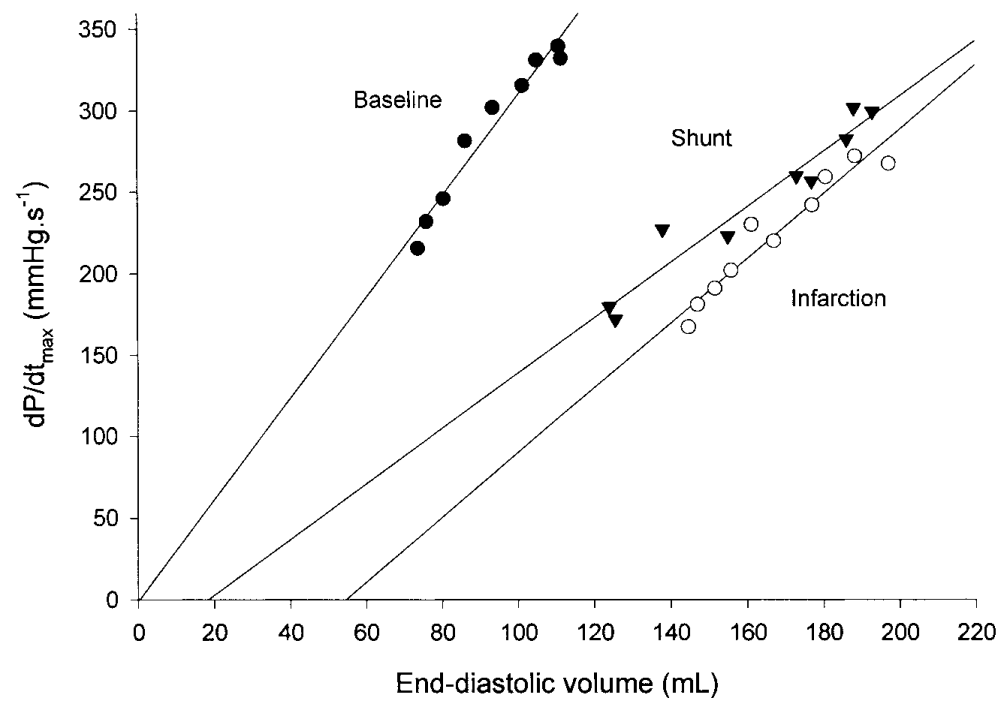

Figure 5. $\mathrm{RV} \mathrm{dP} / \mathrm{dt}_{\text {max }}-\mathrm{EDV}$ at baseline, after ischemia, and after Glenn shunting.

method described by Kono and colleagues ${ }^{15}$ with an iterative algorithm. $\mathrm{V}_{0}$ was initially set to zero, and linear regression of the endsystolic pressure-volume points was performed, generating an ESPVR with a slope and intercept $\mathrm{V}_{0}$. Linear regression was then repeated by using this new value of $\mathrm{V}_{0}$. Within 3 to 6 iterations, $\mathrm{V}_{0}$ would stabilize to a tolerance of $0.01 \mathrm{~mL}$. In a similar fashion the LV end-systolic pressure-length relationship (ESPLR) was calculated by means of segment length instead of ventricular volume.

For each cycle under occlusion, the RV stroke work was calculated as the area of the pressure-volume loop. This was plotted against the end-diastolic volume. Preload recruitable stroke work (PRSW) was derived as the slope of the linear regression between stroke work and end-diastolic volume. ${ }^{16}$ LV segmental stroke work was calculated as the area of the pressure-segment loop. LV PRSW was derived as the slope of the linear regression between segmental stroke work and end-diastolic segment length as follows. ${ }^{17}$

For each beat, the maximum rate of increasing ventricular pressure was calculated and plotted against the end-diastolic volume (EDV). The $\mathrm{dP} / \mathrm{dt}_{\max }$ end-diastolic volume relation and intercept $\left(\mathrm{dP} / \mathrm{dt}_{\max }-\mathrm{EDV}\right)$ is the slope of the linear regression between $\mathrm{dP} / \mathrm{dt}_{\max }$ and EDV. ${ }^{18}$

\section{Calculation of LV Diastolic Function}

The $\mathrm{dP} / \mathrm{dt}_{\text {min }}$ was calculated as the minimum negative $\mathrm{dP} / \mathrm{dt}$ generated by the ventricle. Tau is the time constant of isovolumetric relaxation. ${ }^{19}$ Calculation of the time constant of relaxation is currently the 

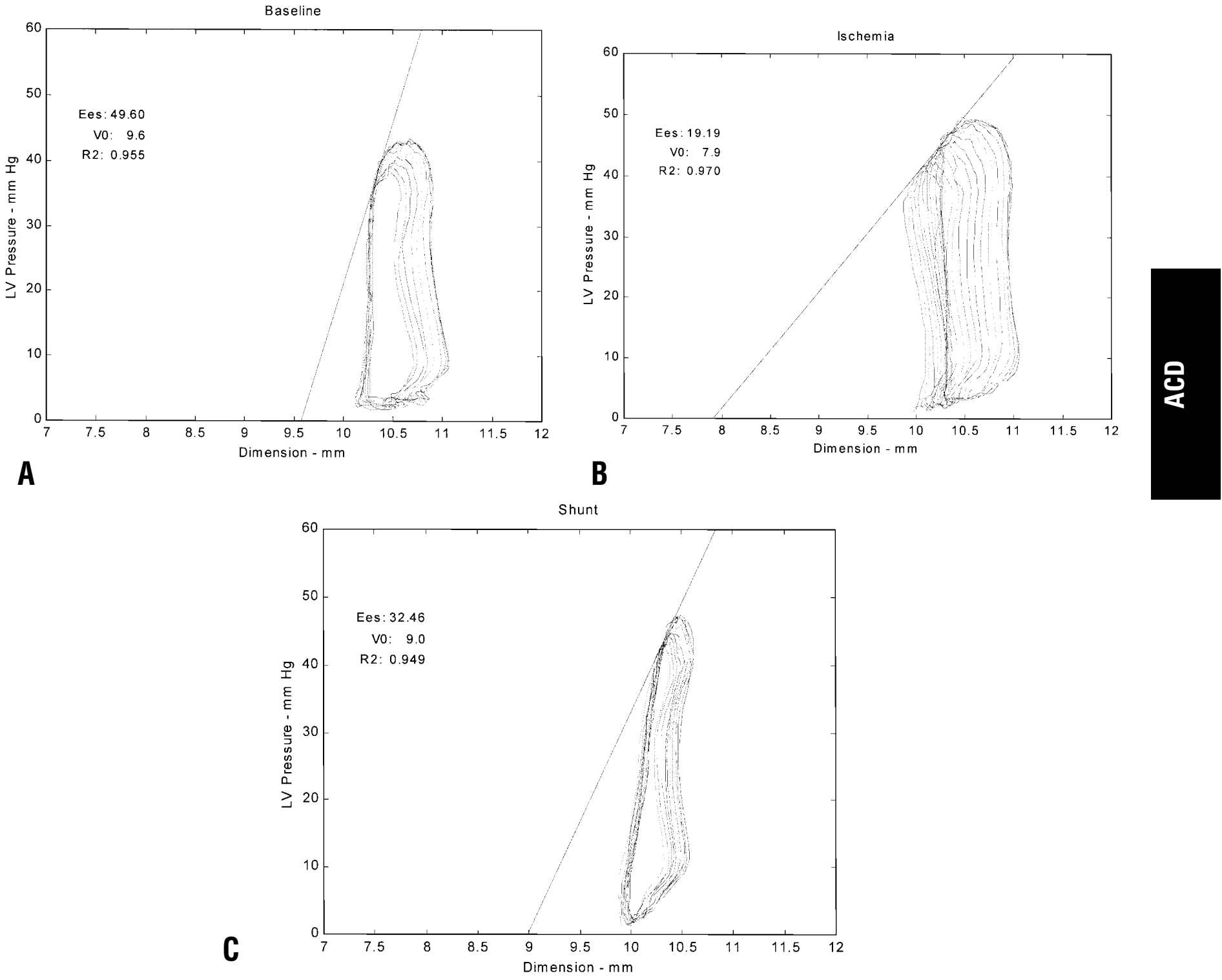

Figure 6. A, LV pressure-segment length loops and ESPLR at baseline. B, LV ESPLR at baseline after ischemia. C, LV ESPLR at baseline after Glenn shunting. Ees, End-systolic elastance; R2, correlation coefficient.

only reliable method of measuring the rate of relaxation. Other parameters, such as isovolumetric relaxation time, are not true representatives of LV relaxation but are dependent on heart rate and peak systolic pressure. ${ }^{20,21}$ The time course of fall in LV pressure from the time of minimum negative $\mathrm{dP} / \mathrm{dt}$ to the level of LV end-diastolic pressure decays exponentially and may be described as follows:

$$
\mathrm{P}=\mathrm{e}^{\mathrm{At}+\mathrm{B}}
$$

where $\mathrm{P}$ is ventricular pressure, $\mathrm{t}$ is time in milliseconds, and $\mathrm{A}$ and $\mathrm{B}$ are constants. This can be expressed as follows:

$$
\ln (\mathrm{P})=\mathrm{At}+\mathrm{B}
$$

where $\ln$ is the natural logarithm.
A logarithmic plot of the data allows the calculation of slope A, a negative number whose units are expressed in seconds. The time constant for LV tau was then defined as -1/A and expressed in milliseconds.

Calculations of gain, parallel conductance, and systolic and diastolic function were performed by means of algorithms developed in our laboratory (Matlab 5.3, The Mathworks Inc, Natick, Mass).

\section{Experiment Protocol}

After surgical preparation and instrumentation, the animal was left undisturbed for 30 minutes to allow stabilization before data collection. All data were collected with the endotracheal tube discon- 

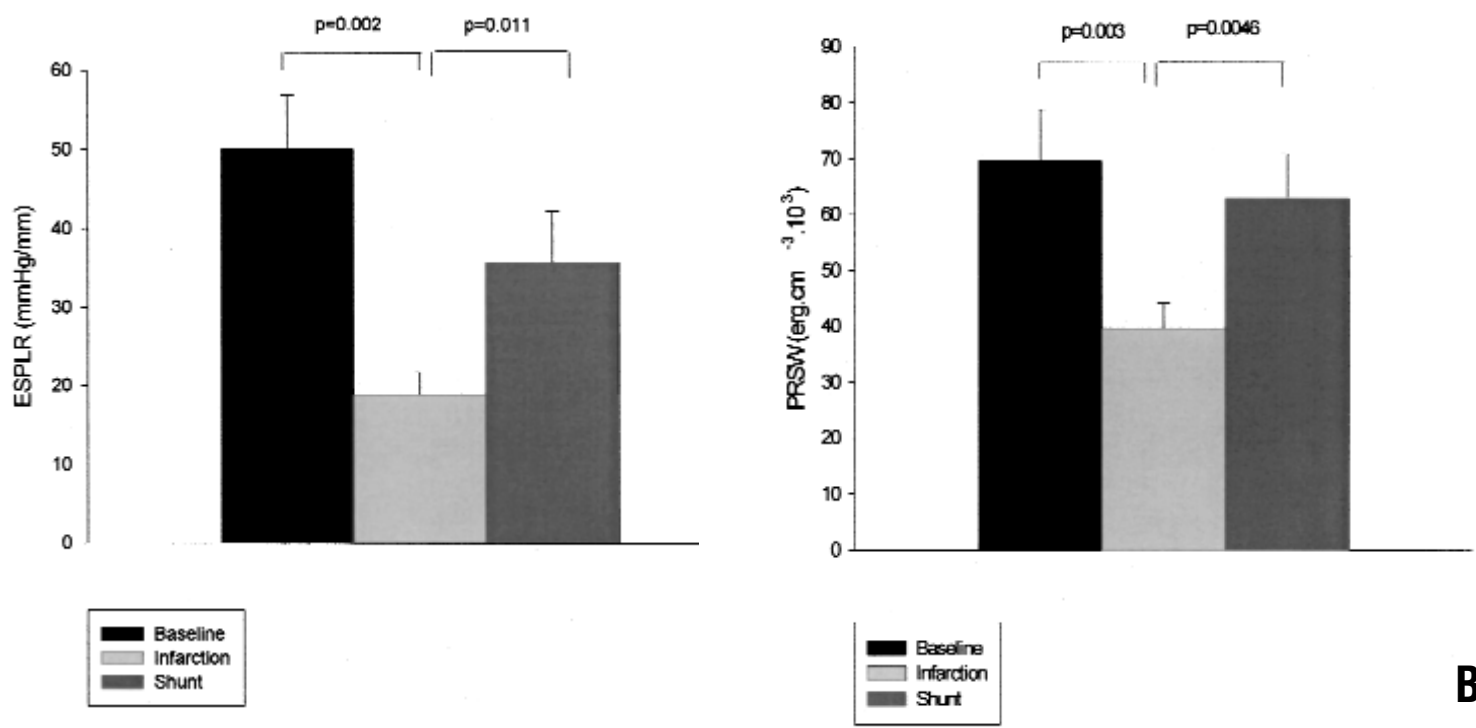

Figure 7. LV ESPLR and PRSW slopes at baseline, ischemia, and after Glenn shunting.

nected from the ventilator. At baseline, steady-state and saline injection data were collected for conductance catheter calibration. Load-independent indices of contraction were derived from occlusion of the IVC.

Isolated RV infarction was induced by means of ligation of the acute marginal coronary arteries. After 30 minutes of stabilization, steady-state and inflow occlusion data were taken.

The cavopulmonary shunt was then opened, and SVC connection to the right atrium was obstructed with vascular clamps, thereby directing all SVC blood to the pulmonary artery. On opening the cavopulmonary shunt, an obvious decrease in the RV size was observed. Increased fluid administration after infarction and shunting was required to maintain systemic blood pressure at baseline levels.

At the end of the experiment, when the animal was killed by means of intraventricular injection of potassium chloride, an autopsy was performed to confirm position of the conductance catheter. The heart was excised, and the shunt was opened to inspect for clots. The hearts were stained to qualitatively analyze the site and magnitude of infarction. In each heart muscle, injury was limited to the RV free wall.

\section{Statistics}

Data were analyzed with the use of SPSS version 8.0 software (SPSS Inc, Chicago, Ill). Data are expressed as means and SDs. Comparisons are made between the baseline and the ischemic state and between the ischemic and shunted state by the paired Student $t$ test.

\section{Results}

All 8 animals survived the period of the study. The hemodynamic insult after RV coronary occlusion was generally well tolerated, allowing repeated inflow occlusions, which are necessary for contractile assessment.

Coronary occlusion produced acute RV dilation with a marked fall in cardiac output from $4.32 \pm 0.6 \mathrm{~L} / \mathrm{min}$ to
$3.10 \pm 0.7 \mathrm{~L} / \mathrm{min}(P=.03) . \mathrm{RV}$ decompression with the cavopulmonary shunt was associated with a significant decrease in RV end-diastolic volume $(169 \pm 70 \mathrm{~mL}$ vs 141 $\pm 66 \mathrm{~mL}$ ) before and after opening of the cavopulmonary shunt. Overall cardiac output was not improved by the cavopulmonary shunt, although there was variation between the animals.

\section{Effect of RV Ischemia and Glenn Shunting on RV Systolic Function}

With the onset of RV ischemia, RV ESPVR shifted in a parallel fashion to the right (Figures 2 and 3) expressed as an increase in $\mathrm{x}$-intercept $\left(\mathrm{V}_{0} ; 33.9 \pm 26 \mathrm{~mL}\right.$ vs $82.4 \pm 19 \mathrm{~mL}$ before and after coronary occlusion, respectively; $P=.002$ ), with a minor and nonsignificant decrease in the slope of the ESPVR $(0.43 \pm 0.2$ vs $0.37 \pm 0.1$ before and after coronary occlusion, respectively; $P=.8$ ). Cavopulmonary shunting and RV decompression produced a significant leftward shift in the ESPVR toward baseline, decreasing $\mathrm{V}_{0}$ from $82.4 \pm$ $19 \mathrm{~mL}$ to $57.0 \pm 22 \mathrm{~mL}$ before and after placement of the cavopulmonary shunt $(P=.001)$. However, a further reduction in the ESPVR slope was observed in the shunted state $(0.37 \pm 0.1$ vs $0.31 \pm 0.1$ before and after cavopulmonary shunting, $P=.07$ ).

By contrast to the response of the ESPVR, the slope of the RV PRSW significantly fell from $16.9 \pm 3 \mathrm{~mm} \mathrm{Hg}$ at baseline to $12.5 \pm 0.5 \mathrm{~mm} \mathrm{Hg}$ after coronary occlusion $(P=$ $.004)$; this was associated with an increase in the $\mathrm{V}_{0}$ from $73.1 \pm 16 \mathrm{~mL}$ to $128.0 \pm 28 \mathrm{~mL}$ before and after ischemia $(P=.001)$ (Figure 4). Cavopulmonary shunting and RV decompression produced a leftward shift in the PRSW relation, with $\mathrm{V}_{0}$ decreasing from $128 \pm 28 \mathrm{~mL}$ with ischemia to $100.2 \pm 29 \mathrm{~mL}$ after placement of the cavopulmonary shunt 

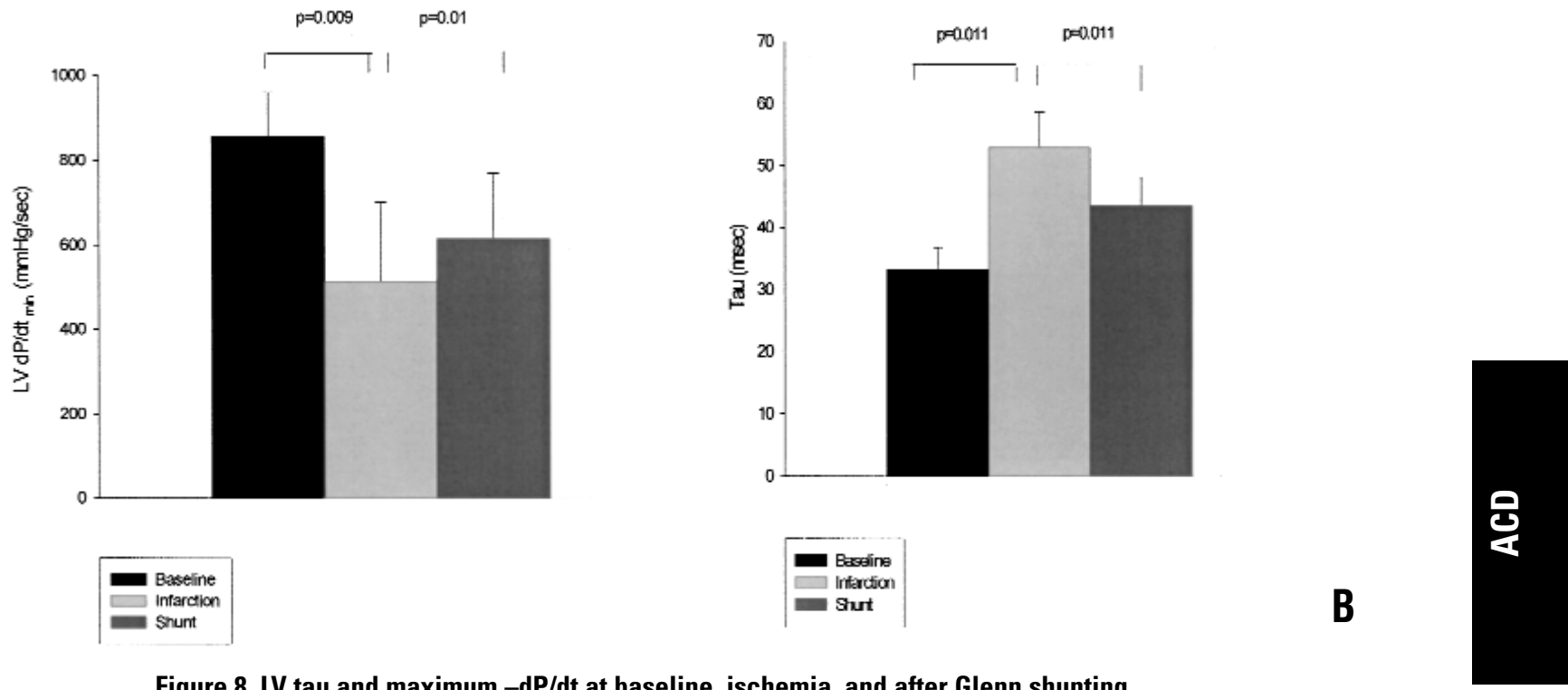

Figure 8. LV tau and maximum - dP/dt at baseline, ischemia, and after Glenn shunting.

$(P=.003)$ and without an increase in the PRSW slope $(12.5$ $\pm 0.5 \mathrm{~mm} \mathrm{Hg}$ vs $11.3 \pm 2.7 \mathrm{~mm} \mathrm{Hg}, P=.25)$.

The third load-independent index of contraction $\left(\mathrm{dP} / \mathrm{dt}_{\max }-\mathrm{EDV}\right)$ responded to ischemia by a reduction in the slope $(8.02 \pm 6$ vs $5.99 \pm 5$ after ischemia, $P=.07)$ and rightward shift in $\mathrm{dP} / \mathrm{dt}_{\max }-\mathrm{EDV}$ position, increasing the $\mathrm{V}_{0}$ from $40.3 \pm 26 \mathrm{~mL}$ at baseline to $64.8 \pm 14 \mathrm{~mL}$ after ischemia $(P=.05)$ (Figure 5). Cavopulmonary shunting and RV decompression did not significantly affect either the $\mathrm{dP} / \mathrm{dt}_{\max }-\mathrm{EDV}$ position, $\mathrm{V}_{0}(64.8 \pm 14 \mathrm{~mL}$ [ischemia] vs $52.6 \pm 16 \mathrm{~mL}$ [after cavopulmonary shunting], $P=.11$ ), or the slope of the relation $(5.99 \pm 5$ [ischemia] vs $5.74 \pm 5$ [after cavopulmonary shunting], $P=.33$ ).

\section{Effect of RV Ischemia and Glenn Shunting on LV Function}

With the onset of RV ischemia, the LV ESPLR slope significantly fell from $50.0 \pm 19 \mathrm{~mm} \mathrm{Hg} / \mathrm{mm}$ at baseline to $18.9 \pm 8 \mathrm{~mm} \mathrm{Hg} / \mathrm{mm}(P=.002)$. With $\mathrm{RV}$ decompression with the Glenn shunt, the slope of the LV ESPLR significantly increased to $35.8 \pm 18 \mathrm{~mm} \mathrm{Hg} / \mathrm{mm}(P=.011)$ (Figures 6 and 7, $A$ ).

Likewise, with RV ischemia, there was a significant decline in the slope of the LV PRSW from $69.6 \pm 26 \mathrm{erg}$. $\mathrm{cm}^{-3} \cdot 10^{3}$ at baseline to $39.7 \pm 13 \mathrm{erg} \cdot \mathrm{cm}^{-3} \cdot 10^{3}(P=$ .003). The LV PRSW slope significantly increased with the shunted state $\left(63 \pm 22 \mathrm{erg} \cdot \mathrm{cm}^{-3} \cdot 10^{3}, P=.005\right)$ (Figure 7 , $B)$. $\mathrm{V}_{0}$ for LV ESPVR and LV PRSW did not change significantly after RV infarction or after Glenn shunting.

LV isovolumetric relaxation was significantly impaired after RV ischemia, with an increase in tau from $33.3 \pm 10 \mathrm{~ms}$ at baseline to $53.0 \pm 16 \mathrm{~ms}$ after ischemia $(P=.015)$ and a reduction in the minimum $-\mathrm{dP}_{\mathrm{dt}}$ min of $-856 \pm 288 \mathrm{~mm} \mathrm{Hg} / \mathrm{s}$ at baseline to $-516 \pm 182 \mathrm{~mm} \mathrm{Hg} / \mathrm{s}$ after $\mathrm{RV}$ ischemia $(P=.009)$. Both these parameters were significantly improved with the Glenn shunt (Table 1 and Figure 8).

\section{Discussion}

The right ventricle was once considered dispensable and considered to act mainly as a passive conduit. ${ }^{22}$ Such conclusions were based on experimental studies in dogs, in which cauterization of the RV free wall was well tolerated and resulted in little hemodynamic compromise. ${ }^{1}$ In addition, the successful outcomes of the Fontan operation, in which adequate pulmonary blood flow can be achieved by means of passive systemic venous pressure without RV contribution, lent further support to this hypothesis. ${ }^{2}$ However, there is now increasing evidence that RV function is an important determining factor in the outcome of many cardiac conditions. Zehender and coworkers ${ }^{23}$ demonstrated that mortality after acute inferior wall infarction is significantly increased with RV involvement, with $26 \%$ versus $64 \%$ complications without and with RV involvement, respectively. RV dysfunction significantly increases mortality after mitral valve replacement ${ }^{24,25}$ and remains a major cause of acute mortality after cardiac transplantation ${ }^{3}$ and LV assist device placement. ${ }^{4}$ Despite this, RV function remains poorly characterized, and the mechanisms by which $\mathrm{RV}$ failure contributes to the hemodynamic insult remain incompletely identified.

\section{Function After RV Ischemia-Induced Dilation}

Previous animal studies have demonstrated that the reduction in cardiac output after RV failure results principally 
TABLE 1. Hemodynamic parameters at baseline, after infarction, and after Glenn shunting

\begin{tabular}{|c|c|c|c|c|c|}
\hline Parameter & Baseline & Infarction & Shunt & $P 1$ & P2 \\
\hline $\mathrm{CO}$ (L/min) & $4.32 \pm 0.62$ & $3.10 \pm 0.67$ & $3.13 \pm 0.39$ & $.03^{*}$ & .24 \\
\hline $\operatorname{LVP}(\mathrm{mm} \mathrm{Hg})$ & $74.4 \pm 8.0$ & $72.1 \pm 9.5$ & $71.8 \pm 8.2$ & .43 & .92 \\
\hline LVEDP (mm Hg) & $6.29 \pm 1.8$ & $9.07 \pm 2.6$ & $7.24 \pm 2.7$ & .05 & .11 \\
\hline $\mathrm{RVP}(\mathrm{mm} \mathrm{Hg})$ & $26.7 \pm 1.7$ & $25.9 \pm 2.3$ & $23.5 \pm 3.4$ & .62 & .32 \\
\hline RVEDP $(\mathrm{mm} \mathrm{Hg})$ & $3.04 \pm 1.8$ & $7.2 \pm 1.5$ & $3.98 \pm 2.3$ & $.0005 \dagger$ & $.006 \dagger$ \\
\hline HR (beats/min) & $89.1 \pm 39$ & $84.8 \pm 22$ & $84.5 \pm 20$ & .41 & .71 \\
\hline RVEDV (mL) & $118.2 \pm 82$ & $169.5 \pm 70$ & $141.4 \pm 66$ & $.0008 \dagger$ & $.0023 \dagger$ \\
\hline RV ESPVR (mm Hg/mL) & $0.43 \pm 0.17$ & $0.37 \pm 0.11$ & $0.31 \pm 0.11$ & .47 & .07 \\
\hline $\mathrm{V}_{0}(\mathrm{~mL})$ & $33.8 \pm 26$ & $82.4 \pm 19$ & $57.0 \pm 22$ & $.002 \dagger$ & $.0012 \dagger$ \\
\hline RV PRSW (mm Hg) & $16.9 \pm 16$ & $12.5 \pm 0.45$ & $11.3 \pm 2.7$ & $.004 \dagger$ & .25 \\
\hline $\mathrm{V}_{0}(\mathrm{~mL})$ & $73.1 \pm 16$ & $128.0 \pm 28$ & $100.2 \pm 29$ & $.001 \dagger$ & $.0003 \dagger$ \\
\hline $\mathrm{RV} \mathrm{dP} / \mathrm{dt}_{\max }-\mathrm{EDV}$ & $8.02 \pm 6.4$ & $5.9 \pm 5$ & $5.7 \pm 4.8$ & .07 & .33 \\
\hline $\mathrm{V}_{0}(\mathrm{~mL})$ & $40.3 \pm 26$ & $64.8 \pm 14$ & $52.6 \pm 16$ & .051 & .11 \\
\hline LV ESPLR (mm Hg/mm) & $50.03 \pm 19$ & $18.9 \pm 7.8$ & $35.8 \pm 18.2$ & $.002 \dagger$ & $.011^{*}$ \\
\hline LV PRSW (erg $\left.\cdot \mathrm{cm}^{-3} \cdot 10^{3}\right)$ & $69.6 \pm 26$ & $39.7 \pm 13$ & $63.0 \pm 22$ & $.003 \dagger$ & $.005 \dagger$ \\
\hline $\mathrm{LV}-\mathrm{dP} / \mathrm{dt}_{\min }(\mathrm{mm} \mathrm{Hg} / \mathrm{s})$ & $-856 \pm 288$ & $-516 \pm 182$ & $-617 \pm 179$ & $.009 t$ & $.01 *$ \\
\hline LV tau (ms) & $33.3 \pm 9.8$ & $53.0 \pm 16$ & $43.5 \pm 12.6$ & $.015^{*}$ & .011 \\
\hline
\end{tabular}

Values are given as means $\pm \mathrm{SD}$. $C O$, Cardiac output; $L V P$, peak LV pressure; $L V E D P$, LV end-diastolic pressure; $R V P$, peak RV pressure; RVEDP, RV end-diastolic pressure; $H R$, heart rate; RVEDV, RV end-diastolic volume; ESPVR, end-systolic pressure-volume relationship slope; $\mathrm{V}_{0}+\mathrm{intercept} P R S W$, preloadrecruitable stroke work; $d P / d t_{\max }-E D V$, slope of relation between maximum $\mathrm{dP} / \mathrm{dt}$ and $E D V ; E S P L R$, end-systolic pressure-length relationship; $L V-d P / d t_{\text {min }}$ $\mathrm{LV}$ minimum - $\mathrm{dP} / \mathrm{dt}$; $L V$ tau, time constant of $\mathrm{LV}$ isovolumetric relaxation; $P 1$, comparison of baseline and infarction; $P 2$, comparison of infarction and shunted state.

${ }^{*} P<.05$.

$\dagger P<.01$.

from diastolic interaction with reduced LV filling and compliance. In a dog model, Goldstein and associates ${ }^{10}$ showed that elevated intrapericardial pressure after RV infarction reduced LV preload and cardiac output. Calvin ${ }^{25 a}$ demonstrated that RV infarction reduced cardiac output by $23 \%$ and stroke volume by $30 \%$ and decreased LV size, as determined by end-diastolic segment length. Goto and colleagues, ${ }^{8}$ using a dog model, demonstrated that RV infarction was associated with a leftward and upward shift of the LV end-diastolic pressure-volume relationship without changing LV myocardial performance.

What is less clear is the effect of RV dilation on LV systolic function. Previous studies have concluded that LV systolic performance is unaffected by RV ischemia on the basis of insignificant changes in aortic flow, peak $\mathrm{dP} / \mathrm{dt}$, and percentage of systolic shortening. ${ }^{8}$ However, such parameters fail to evaluate the LV contractile state independent of the loading conditions. In our study, LV systolic function was determined by using ESPLR and PRSW, indices that are load independent, and distinguishing changes in contractility from changes in the preload and afterload. We found that the LV contractility was significantly impaired after RV infarction, as indicated by decreases in the slopes of ESPLR and PRSW. These results are in agreement with those of the study by Brookes and associates, ${ }^{9}$ in which LV systolic function was impaired after RV ischemia in pigs. However, in that study RV ischemia was induced by occlusion of the main right coronary artery. In pigs the right coronary artery is dominant in the majority of animals and supplies the inferior interventricular septum and a variable amount of the inferior LV wall. ${ }^{26}$ It is possible that the LV contractile deficit they observed was the direct result of the interventricular septum-inferior LV wall. By contrast, in the present study infarction was limited to the RV free wall, as demonstrated by postmortem staining.

Although LV compliance is decreased with RV ischemia, the effect on LV relaxation has not been previously studied. We identified that LV isovolumetric relaxation was significantly impaired after RV infarction with an increase in tau, the time constant of isovolumetric relaxation, and increase in minimum $-\mathrm{dP} / \mathrm{dt}$. Tau is a measure of the active and energy-dependent process of ventricular relaxation and involves adenosine triphosphatase-mediated removal of calcium from the contractile proteins and sequestration within the sarcoplasmic reticulum. This process is prolonged in ischemia or acute infarction and is unaffected by the loading conditions. It is possible that the RV ischemic dilation with associated distortion in LV geometry has a direct effect on active relaxation in addition to the contractile deficit.

\section{RV Systolic Function After Acute RV Free-Wall Infarction}

Despite acknowledging the importance of RV function, quantifying contractility has been limited because of difficulties in measuring continuous RV volume. Unlike the left ventricle, the right ventricle has a densely trabeculated inter- 
nal structure and a cavity shape that cannot be adequately described with a simple mathematical model. Methods of RV volume determination, such as shell subtraction and sonomicrometry, derive volume by measuring a series of ventricular dimensions and assuming constant ventricular geometry during the cardiac cycle and changes in loading conditions. Such techniques may be vulnerable in conditions of regional wall abnormalities or major shifts in septal position, in which significant changes in ventricular cavity geometry would be expected. In this study, RV volume was measured by the conductance catheter technique. Although the determination of absolute volume by conductance requires complex calibration procedures (parallel conductance and gain), the method has proven reliable for LV volume determination and has been used extensively in clinical and experimental studies. Although experience is less in the right ventricle, it has been used in animal studies ${ }^{27}$ and clinical studies involving adult ${ }^{28}$ and pediatric populations. ${ }^{29}$ In this study, an alternative method of RV volume measurement was not used; however, during the calculation of gain, ventricular volumes derived by conductance were compared with the integrated pulmonary artery flow during ejection, and strong linear relationships were consistently apparent.

In this study, RV contractility was determined by using 3 indices of load-independent contraction: ESPVR, PRSW, and $\mathrm{dP} / \mathrm{dt}_{\max }$-EDV. The ESPVR was developed by Suga and Shoukas ${ }^{30}$ on the basis of the time-varying elastance model of LV contraction; the relationship is typically linear over a wide range of loading conditions and sensitive to the inotropic state. Maughan and coworkers ${ }^{31}$ showed that the assumptions that underlie the time-varying elastance model of Suga and colleagues ${ }^{30}$ are generally valid for the canine right ventricle, and in the present study we found all 3 indices to be highly linear under the changing load conditions. Furthermore, recent studies have shown that these contractile indices are sensitive to the inotropic state of the right ventricle and have thus been used to quantify RV contraction in animal ${ }^{27,32}$ and clinical ${ }^{27}$ studies. Despite this, little information exists regarding the effect of isolated RV ischemia-infarction on these contractile indices. We found that after free-wall RV infarction, the ESPVR was displaced rightward, with a significant increase in $V_{0}$ and minimal changes in slope. This is similar to the effect of regional ischemia on the LV ESPVR. ${ }^{33}$ Although both RV PRSW and $\mathrm{dP} / \mathrm{dt}_{\text {max }}$-EDV were associated with rightward shift and a decrease in gradient after infarction, PRSW was more consistent. Therefore, PRSW appears superior to ESPVR in assessing the contractile state in the RV; this is in agreement with other reports. ${ }^{32}$ This may be due to the difficulties in defining the point of end-systole in the RV, and less error was associated with the measurement because the whole loop is used in PRSW determination rather than a single point, as in the cycle with ESPVR.

\section{Effect of Glenn Shunting on Ventricular Function}

Institution of the cavopulmonary shunt reduced RV volume and significantly improved the LV systolic impairment observed with the RV infarction. In addition, LV isovolumetric relaxation and minimum $-\mathrm{dP} / \mathrm{dt}$ were also improved by using the shunt. Studies have demonstrated that after RV infarction, LV systolic function and compliance can be improved by means of pericardiotomy. ${ }^{9}$ In addition, volume offloading by means of lower body suction can improve LV filling by normalizing septal position in patients with congestive heart failure. ${ }^{34}$ These studies emphasize the importance of RV dilation and ventricular interaction in the role of low cardiac output after RV ischemia. The mechanism by which the cavopulmonary shunt effectively improves LV performance is likely to be a reduction in the RV volume with restoration of interventricular septal position and LV cavity shape. We did not observe an improvement in RV contractility; the slopes of RV ESPVR, PRSW, and $\mathrm{dP} / \mathrm{dt}_{\text {max }}$-EDV did not change after the Glenn shunt, although leftward shifts in the contractile indices were observed.

\section{Study Limitations}

Despite the improvement in LV performance after placement of the Glenn shunt, overall cardiac output did not significantly increase, although there was a variable response between animals. It is possible that preload to the left ventricle remained low or possibly diminished after the Glenn shunt was instituted. A similar response is widely recognized in children after Glenn shunting or complete Fontan procedures; initial systemic venous may be high with low cardiac output, and with time (often 4-6 hours after the operation), pulmonary blood flow and cardiac output increase and systemic venous pressures decrease. In our preparation the hemodynamic data were usually acquired within 30 minutes of institution of the shunt; perhaps allowing more time for the preparation to accommodate to the surgical reconstruction may have improved the cardiac output. Furthermore, measurement of simultaneous SVC and right and left atrial pressures together with shunt flow and RV output would allow better manipulation of the systemic venous pressures to optimize pulmonary blood flow and LV diastolic filling. Finally, the use of negative pressure ventilation or spontaneous respiration may also improve pulmonary blood flow and cardiac output by decreasing pulmonary vascular resistance.

\section{Clinical Implications}

If LV geometry has a major influence on contractility and relaxation, this may explain why volume loading in the context of RV dilation may not improve the cardiac output. Pericardiotomy improves the LV contractility and compliance after RV ischemia by restoring the geometric arrange- 
ments. This may explain the hemodynamic benefit of leaving the sternum open in the early postoperative period in patients with RV dysfunction after cardiopulmonary bypass.

Normalizing the septal position and LV geometry may be achieved by limiting RV dilation with a reduction in preload. Creation of an atrial septal defect is commonly used to offload the right ventricle in patients in whom the RV function may be temporally impaired after right ventriculotomy or extensive muscle resection of the RV outflow tract in the repair of tetralogy of Fallot. Although this will be associated with a degree of cyanosis, it is well tolerated in children who have adapted to a cyanotic physiology. By contrast, in the adult population creation of an atrial septal defect for RV dysfunction after cardiopulmonary bypass has been associated with high mortality. The cyanosis is poorly tolerated, and the increasing acidosis exacerbates the myocardial dysfunction. The advantage of a Glenn circuit is that RV preload and dilation are reduced, and the oxygen saturations are still maintained. However, the pulmonary vascular resistance and LV end-diastolic pressure must be low to achieve adequate shunt flow.

\section{Conclusions}

We have shown in this study that LV contractility and relaxation are adversely affected by RV ischemia, which is limited to the RV free wall. We believe this contributes significantly to the hemodynamic deterioration that follows RV ischemia and results from distortion of the LV cavity geometry and changes in septal position arising from the RV dilation. Limiting the RV dilation by reducing preload by means of a Glenn shunt restored both the LV contractile and diastolic deficit. This may be a therapeutic option in patients who sustain primary RV failure with normal pulmonary artery pressures after cardiopulmonary bypass.

\section{References}

1. Starr I, Meade R. The absence of conspicuous increments of venous pressure after severe damage to the right ventricle in the dog, with discussion of the relation between clinical congestive failure and heart disease. Am Heart J. 1943;26:291-301.

2. Fontan F, Baudet E. Surgical repair of tricuspid atresia. Thorax. 1971;26:240-8.

3. Szabo G, Sebening C, Hagl C, Tochtermann U, Vahl CF, Hagl S. Right ventricular function after brain death: response to an increased afterload. Eur J Cardiothorac Surg. 1998;13:449-59.

4. Farrar DJ, Compton PG, Hershon JJ, Fonger JD, Hill JD. Right heart interaction with mechanically assisted left heart. World J Surg. 1985;9:89-102.

5. Moran JM, Opravil M, Gorman AJ, Rastegar H, Meyers SN, Michaelis LL. Pulmonary artery balloon counterpulsation for right ventricular failure. Ann Thorac Surg. 1984;38:254-9.

6. Karagoz HY, Tasdemir O, Yatut C, Bayazit K. Atrial septal defect for right ventricular failure [letter]. Ann Thorac Surg. 1988;45:350-1.

7. Goldstein JA, Barzilai B, Rosamond TL, Eisenberg PR, Jaffe AS. Determinants of hemodynamic compromise with severe right ventricular infarction. Circulation. 1990;82:359-68.

8. Goto Y, Yamamoto J, Saito M, Haze K, Sumiyoshi T, Fukami K, et al. Effect of right ventricular ischemia on left ventricular geometry and the end-diastolic pressure-volume relationship in the dog. Circulation. 1985;72:1104-14.

9. Brookes C, Raven H, White P, Moeldrup U, Oldershaw P, Redington A. Acute right ventricular dilation in response to ischemia significantly impairs left ventricular systolic performance. Circulation. 1999; 100:761-7.

10. Goldstein JA, Vlahakes GJ, Verrier ED, Schiller NB, Tyberg JV, Ports TA, et al. The role of right ventricular systolic dysfunction and elevated intrapericardial pressure in the genesis of low output in right ventricular infarction. Circulation. 1982;65:513-22.

11. Kreutzner C, Kreutzner GOA, Conejores W, Roman MI, Vasquez H, Schlichter AJ, et al. Experience with the one and a half ventricle repair. J Thorac Cardiovasc Surg. 1999;117:662-8.

12. Hetzer R, Nagdyman N, Ewert P, Weng YG, Meskhisvili VA, Berger $\mathrm{F}$, et al. A modified repair technique for tricuspid incompetence in Ebstein's anomaly. J Thorac Cardiovasc Surg. 1998;115:857-68.

13. Swindle MM, Horneffer PJ, Gardner TJ, Gott VL, Hall TS, Stuart RS, et al. Anatomic and anesthetic considerations in experimental cardiopulmonary surgery in swine. Lab Anim Sci. 1986;36:357-61.

14. Baan J, Steendijk P, Koops J. Calibration and application of the conductance catheter for ventricular volume measurement. Automedica. 1989;11:357-65.

15. Kono A, Maughan WL, Sunagawa K, Hamilton K, Sagawa K, Weisfeldt ML. The use of left ventricular end-ejection pressure and peak pressure in the estimate of the end-systolic pressure-volume relationship. Circulation. 1984;70:1057-65.

16. Glower DD, Spratt JA, Snow ND, Kabas JS, Davis JW, Olsen CO, et al. Linearity of the Frank-Starling relationship in the intact heart: the concept of preload recruitable stroke work. Circulation. 1985;71:9941009.

17. Glower DD, Kabas JS, Davis JW, Rankin JS. Quantification of regional myocardial dysfunction after acute ischemic injury. Am J Physiol. 1988;24:H85-93.

18. Little $\mathrm{W}$. The left ventricular $\mathrm{dP} / \mathrm{dtmax}-$ end-diastolic volume relation in closed-chest dogs. Circ Res. 1985;56:808-15.

19. Weiss J, Frederiksen JW, Weisfeldt ML. Hemodynamic determinants of the time course of fall in canine left ventricular pressure. J Clin Invest. 1976;58:751-60.

20. Yellin EL, Yoran C. Left ventricular relaxation in the filling and nonfilling intact canine heart. Am J Physiol. 1986;250:620-9.

21. Paulus WJ, Vantrimpont PJ, Rousseau MF. Diastolic function of the nonfilling human left ventricle. J Am Coll Cardiol. 1992;20:1524-32.

22. Sade R, Castaneda AR. The dispensable right ventricle. Surgery. 1975;77:624-31.

23. Zehender M, Kasper W, Kauder E, Schonthaler M, Geibel A, Olschewski M, et al. Right ventricular infarction as an independent predictor of prognosis after acute inferior myocardial infarction. $N$ Engl J Med. 1993;328:981-8.

24. Ferrazzi P, McGriffin DC, Kirklin JW, Blackstone EH, Bourge RC. Have the results of mitral valve replacement improved? J Thorac Cardiovasc Surg. 1986;92:186-97.

25. Cummings R, Hull-Ryde E, Schumacher D. Differential susceptibility of the left and right ventricles and interventricular septum to irreversible injury during total ischemia. Surg Forum. 1986;37:295-7.

25a. Calvin JE. Optimal right ventricular filling pressures and the role of pericardial constraint in right ventricular infarction in dogs. Circulation. 1991;84:852-61.

26. Brookes H, Al-Sadir J. Right ventricular performance during ischemia: an anatomical and hemodynamic analysis. Am J Physiol. 1977;233:H500-13.

27. Dickstein ML, Yano O, Spotnitz HM, Burkhof D. Assessment of right ventricular contractile state with the conductance catheter technique in the pig. Cardiovasc Res. 1995;29:820-8.

28. Brookes CI, White PA, Bishop AJ, Oldershaw PJ, Redington AN, Moat NE. Validation of a new intraoperative technique to evaluate load-independent indices of right ventricular performance in patients undergoing cardiac surgery. J Thorac Cardiovasc Surg. 1998;116:468-76.

29. White PA, Chaturvedi RR, Bishop AJ, Brookes CI, Oldershaw PJ, Redington AN. Does parallel conductance vary during systole in the human right ventricle? Cardiovasc Res. 1996;32:901-8. 
30. Suga H, Sagawa K, Shoukas AA. Load independence of the instantaneous pressure-volume ratio of the canine left ventricle and the effects of epinephrine and heart rate on the ratio. Circ Res. 1973;32:314-22.

31. Maughan WL, Shoukas AA, Sagawa K, Weisfeldt ML. Instantaneous pressure-volume relationship of the canine right ventricle. Circ Res. 1979;44:309-15

32. Karunanithi MK, Michnicwicz J, Copeland SE, Feneley MP. Right ventricular preload recruitable stroke work, end-systolic pressure volume, and $\mathrm{dP} / \mathrm{dtmax}$-end-diastolic volume relations compared as indexes of right ventricular contractile performance in conscious dogs. Circ Res. 1992;70:1169-79.

33. Sunagawa K, Maughan WL, Sagawa K. Effect of regional ischemia on the left ventricular end-systolic pressure volume relationship of isolated canine hearts. Circ Res. 1983;52:170-8.
34. Atherton JJ, Moore TD, Lele SS, Thomson HL, Galbraith AJ, Belenkie I, et al. Diastolic ventricular interaction in chronic heart failure. Lancet. 1997;349:1720-4.

\section{Discussion}

Dr Robert W. Emery (Minneapolis, Minn). Did you leave the vena cava open during the shunt opening, or did you clamp it to divert the flow?

Dr Danton. The cava was clamped, and the azygos vein was tied off. 